\title{
On the Minimax Robustness of the Uniform Transmission Power Strategy in MIMO Systems
}

\author{
Shuangqing Wei, Dennis Goeckel
}

\begin{abstract}
In this letter, it is shown that the uniform power allocation across transmit antennas is optimal in the sense that this strategy will maximize the minimum average mutual information of a multiple-input-multiple-output (MIMO) system across the class of any arbitrary correlated fading channels, with constraints on the the total fixed transmit power $\left(P_{Q}\right)$, total power of the fades at the transmitter side $\left(P_{T}\right)$, and total power of the fades at the receiver side $\left(P_{R}\right)$, if the channel state information (CSI) is perfectly known at the receiver side only.
\end{abstract}

\section{INTRODUCTION}

Employing multiple antennas at both the transmitter and receiver of a communication system operating over a narrowband wireless communications channel can significantly increase the Shannon capacity in the scenario with independent fading [1] [2], or correlated fading [3] across different antenna pairs. Consider a MIMO system with $n_{T}$ transmit antennas and $n_{R}$ receive antennas, and let $H_{i, j}$ be the fading coefficient between the $j$ th transmit antenna and the $i$ th receive antenna. Assume the receiver has the perfect channel state information (CSI), while the transmitter does not have CSI. If $H_{i, j}$ and $H_{k, l}$, for any disparate pairs $i, j$ and $k, l$, are independent complex Gaussian random variables, it is shown in [2] that the optimal strategy to maximize the average mutual information of such a MIMO system is to transmit statistically independent identically distributed complex Gaussian codewords across $n_{T}$ antennas with equal power $P_{Q} / n_{T}$, where $P_{Q}$ is the total transmit power. If $H_{i, j}$ and $H_{k, l}$ are correlated, and in addition to lacking CSI, the transmitter is also ignorant of values of the correlations, it is assumed without rigorous justification in [3]-[4] that in order to maximize the average mutual information of a MIMO system under correlated fades, the uniform power distribution across transmit antennas will "naturally" be employed [3]. In [5], assuming that channel can play the role of a malicious nature by altering fading values $H_{i, j}$ to perform an inverse water-filling, it has been shown that the uniform power allocation strategy can maximize the minimum average mutual information of a MIMO system. However, under the constraints only on the variances of the fading values, when the channel can only set correlation properties of the fades, the optimality of the uniform power allocation strategy is still an open problem. In this paper, by following the line of the work [5], this strategy will be demonstrated to be minimax robust [6], which maximizes the minimum average mutual information of a MIMO system with arbitrary correlated fades under certain power constraints.

\section{SySTEM MOdEL}

Throughout the paper, the following notations will be used: $I_{N}$ for the $N \times N$ identity matrix, $A^{\dagger}$ for transpose conjugate of the matrix $A, A^{*}$ for conjugate of the matrix $A, \operatorname{det}(A)$ for determinant of the square matrix $A, A^{\prime}$ for transpose of the matrix $A$, and $\underline{X}$ for column vector.

The discrete-time equivalent system model is given by: $\underline{Y}=H \underline{X}+\underline{Z}$, where $\underline{X}$ is an $n_{T} \times 1$ column vector whose $j$ th component represents the signal transmitted by the $j$ th antenna. Similarly, the received signal and received noise are represented by $n_{R} \times 1$ complex column vectors, $\underline{Y}$ and $\underline{Z}$, respectively. The noise vector $\underline{Z}$ is an additive white Gaussian random vector, whose entries $\left\{Z_{i}, i=1, \ldots, n_{R}\right\}$ are i.i.d circularly symmetric complex Gaussian random variables with mean zero and unit variance, thus $Z_{i} \sim \tilde{N}(0,1)$.

It is assumed here that the total average power transmitted across the $n_{T}$ transmit antennas is fixed, i.e. $E\left[\sum_{k=1}^{N}\left|X_{k}\right|^{2}\right]=P_{Q}$. Entries of the channel fading matrix $H$ are assumed to be circularly symmetric complex Gaussian random variables with zero mean, and thus a Rayleigh fading channel is being assumed. Constraints on variances of $H_{i, j}$ will be described below. Per above, it is assumed that the transmitter has neither knowledge of the entries of $H$ nor knowledge of the correlation statistics of the entries, but that the receiver has perfect knowledge of $H_{i, j}$. Hence, as in [2], if the input vector $\underline{X}$ is a proper complex Gaussian random vector, whose covariance matrix is $E\left[\underline{X} \cdot \underline{X}^{\dagger}\right]=Q$, the mutual information $\Phi(Q)$ of this MEA system (conditioned on $H$ ) is $\Phi(Q)=\log _{2} \operatorname{det}\left(I_{n_{R}}+H \cdot Q \cdot H^{\dagger}\right)$ bps/Hz.

It is assumed that the covariance matrix of the random variables $H_{i, j}$ has the following general covariance structure, as described in [3]: $E\left[H_{i, k} H_{j, l}^{*}\right]=\Psi_{k, l}^{T} \Psi_{i, j}^{R}$, where $\Psi^{T}$ and $\Psi^{R}$ are $n_{T} \times n_{T}$ and $n_{R} \times n_{R}$ covariance matrices generated by the transmit and receive antennas, respectively. As in [3], the matrix $H$ can be factored in the form $H \stackrel{\mathcal{D}}{=}\left(\Psi^{R}\right)^{\frac{1}{2}} W\left(\Psi^{T}\right)^{\prime \frac{1}{2}}$, where the entries of $W$ are i.i.d with $\tilde{N}(0,1)$, and $x \stackrel{\mathcal{D}}{=} y$ means random variables $x$ and $y$ have the same distribution.

Our goal here is to find the minimax robust $Q_{0}$ [6], under the constraint of $\operatorname{Tr}(Q)=P_{Q}, \operatorname{Tr}\left(\Psi^{T}\right)=P_{T}$ and $\operatorname{Tr}\left(\Psi^{R}\right)=P_{R}$, where $\operatorname{Tr}(A)$ is the trace of matrix $A$ [7], to maximize the infimum average mutual information $E[\Phi(Q)]$, i.e.,

$$
Q_{0}=\arg \max _{Q \in S_{Q}} \inf _{\Psi^{R} \in S_{R}, \Psi^{T} \in S_{T}} E[\Phi(Q)]
$$

where $S_{Q}=\left\{Q: \operatorname{Tr}(Q)=P_{Q}\right\}, \quad S_{T}=$ $\left\{\Psi^{T}: \operatorname{Tr}\left(\Psi^{T}\right)=P_{T}\right\}$ and $S_{R}=\left\{\Psi^{R}: \operatorname{Tr}\left(\Psi^{R}\right)=P_{R}\right\}$, are the sets of non-negative definite matrices with the constraint 
of fixed trace, which are all convex sets. The expectation $E[\cdot]$ is over the statistical distribution of the fading entries of $H$ under the given correlation matrices $\Psi^{T}, \Psi^{R}$ and $Q$. The trace constraints for channel correlation matrices $\Psi^{T}$ and $\Psi^{R}$ imply that the total power of the fades caused by scatterings around transmit and receive antennas are fixed as $P_{T}$ and $P_{R}$, respectively.

\section{THEOREM AND PROOF}

Theorem 1: The minimax robust solution to (1) is $Q_{0}=$ $P_{Q} I_{n_{T}} / n_{T}$, and

$\max _{Q \in S_{Q}} \inf _{\Psi^{R} \in S_{R}, \Psi^{T} \in S_{T}} E[\Phi(Q)]=E\left[\log _{2}\left(1+P_{T} P_{R} \frac{P_{Q}}{n_{T}} y\right)\right]$,

where $y$ is an exponentially distributed random variable with unit mean.

Proof:

By singular value decomposition (SVD) [7], it can be shown that $Q=U_{Q} D_{Q} U_{Q}^{\dagger}, \Psi^{T}=U_{T} D_{T} U_{T}^{\dagger}$, and $\Psi^{R}=U_{R} D_{R} U_{R}^{\dagger}$, where $U_{Q}, U_{T}$ and $U_{Q}$ are unitary matrices, and $D_{Q}, D_{T}$ and $D_{R}$ are diagonal matrices whose diagonal entries $\left\{\lambda_{k}^{Q}\right\}$, $\left\{\lambda_{k}^{T}\right\}$ and $\left\{\lambda_{k}^{R}\right\}$ are the eigenvalues of $Q, \Psi^{T}$ and $\Psi^{R}$, respectively, in a decreasing order.

By substituting $H \stackrel{\mathcal{D}}{=}\left(\Psi^{R}\right)^{\frac{1}{2}} W\left(\Psi^{T}\right)^{\frac{1}{2}}$ into $\Phi(Q)$, and recognizing that for any unitary matrices $U$ and $V, U W V^{\dagger}$ has the same statistical distribution as $W$ [2], where entries of $W$ are independently distributed as $\tilde{N}(0,1)$, it can be shown that

$$
\begin{aligned}
E[\Phi(Q)]= & E\left[\operatorname { l o g } _ { 2 } \operatorname { d e t } \left(I_{n_{R}}+\right.\right. \\
& \left.\left.D_{R}^{\frac{1}{2}} W D_{T}^{\frac{1}{2}} V D_{Q} V^{\dagger} D_{T}^{\frac{1}{2}} W^{\dagger} D_{R}^{\frac{1}{2}}\right)\right] \\
= & E\left[\log _{2} \operatorname{det}\left(I_{n_{R}}+D_{R}^{\frac{1}{2}} W B_{2} W^{\dagger} D_{R}^{\frac{1}{2}}\right)\right] \\
= & E\left[\log _{2} \operatorname{det}\left(I_{n_{R}}+D_{R}^{\frac{1}{2}} W D_{B} W^{\dagger} D_{R}^{\frac{1}{2}}\right)\right]
\end{aligned}
$$

where $V=U_{T}^{\prime} U_{Q}$ is a unitary matrix, $B_{1}=D_{T}^{\frac{1}{2}} V D_{Q}^{\frac{1}{2}}$, and $B_{2}=B_{1} B_{1}^{\dagger}$. $D_{B}$ is the diagonal matrix whose diagonal entries are eigenvalues of $B_{2}, \lambda_{k}^{B}$, in a decreasing order, with $\sum_{k=1}^{n_{T}} \lambda_{k}^{B}=\operatorname{Tr}\left(B_{2}\right)=P_{B}$.

Letting $\alpha_{k}=\lambda_{k}^{B} / P_{B}$,

$$
\begin{aligned}
E[\Phi(Q)] & \stackrel{(a)}{\geq} \sum_{k=1}^{n_{T}} \alpha_{k} E\left[\log _{2}\left(1+P_{B} \sum_{j=1}^{n_{R}} \lambda_{j}^{R}\left|w_{j, k}\right|^{2}\right)\right] \\
& \stackrel{(b)}{=} E\left[\log _{2}\left(1+P_{B} \sum_{j=1}^{n_{R}} \lambda_{j}^{R}\left|w_{j, 1}\right|^{2}\right)\right] \\
& \stackrel{(c)}{\geq} E\left[\log _{2}\left(1+P_{B} P_{R}\left|w_{1,1}\right|^{2}\right)\right] \\
& \stackrel{(d)}{\geq} E\left[\log _{2}\left(1+P_{T} P_{R} \lambda_{m i n}^{Q}\left|w_{1,1}\right|^{2}\right)\right]
\end{aligned}
$$

where the inequality (a) is due to the concavity of the function $\log \operatorname{det} A$ on the convex set of non-negative Hermitian matrices [7, pp. 466], as well as the representation of $D_{B}=P_{B} \sum_{k=1}^{n_{T}} \alpha_{k} \underline{e}_{k} \underline{e}_{k}^{\dagger}$, where $\underline{e}_{k}$ is a column vector with 1 as its $k$ th component and 0 's elsewhere. Equality (b) is because the sum term is identically distributed. Inequality (c) is due to the concavity of the function $\log x$ over the region $x>0$, and $\sum_{j=1}^{n_{R}} \lambda_{j}^{R}=P_{R}$. Inequality (d) is because $P_{B}=\sum_{i=1}^{n_{T}} \sum_{j=1}^{n_{T}} \lambda_{i}^{Q} \lambda_{j}^{T}\left|v_{i, j}\right|^{2} \geq P_{T} \lambda_{m i n}^{Q}$, where the last step is due to $\lambda_{i}^{Q} \geq \lambda_{m i n}^{Q}, \forall i$, and $\sum_{i=1}^{n_{T}}\left|v_{i, j}\right|^{2}=1$, since $V$ is a unitary matrix. Therefore, for any covariance matrices $Q$, $\Psi^{T}$ and $\Psi^{R}, E[\Phi(Q)]$ can be lower bounded as that in (5).

For any given transmission strategy $Q$, the lower bound in (5) can be achieved by setting the eigenvalue $\lambda_{1}^{T}=P_{T}, \lambda_{k}^{T}=$ $0, k=2, \ldots, n_{T}$, and $\lambda_{1}^{R}=P_{R}, \lambda_{j}^{R}=0, j=2, \ldots, n_{R}$, with $U_{T}=U_{Q}^{*}$ and $U_{R}$ any arbitrary unitary matrix. Thus, the worst case of channel puts all of its energy at the transmission side in the direction of the weakest eigenvector of $Q$, while the energy of the channel at the receiver side is concentrated in any eigen-direction. Thus,

$\inf _{\Psi^{R} \in S_{R}, \Psi^{T} \in S_{T}} E[\Phi(Q)]=E\left[\log _{2}\left(1+P_{T} \lambda_{\min }^{Q} P_{R}\left|w_{1,1}\right|^{2}\right)\right]$.

Our goal is in (1) to find the minimax robust $Q$, and since $\sum_{k=1}^{n_{T}} \lambda_{k}^{Q}=P_{Q}, \lambda_{\min }^{Q} \leq P_{Q} / n_{T}$ (otherwise, the condition of the fixed total transmission power will be violated), the upper bound of (6) can be achieved by setting $Q=P_{Q} I_{n_{T}} / n_{T}$, which is exactly the transmission strategy of the uniform power distribution across transmit antennas. Hence, (2) is proven to be true.

It can be observed that if $Q_{0}=P_{Q} I_{n_{T}} / n_{T}$, an arbitrary choice of the eigenvectors of $\Psi_{0}^{T}$ and $\Psi_{0}^{R}$ will minimize the mutual information as long as only one eigenvalue of each is non-zero. This set of $\left(Q_{0}, \Psi_{0}^{T}, \Psi_{0}^{R}\right)$ is not a saddle point [6], since given such $\left(\Psi_{0}^{T}, \Psi_{0}^{R}\right)$, the uniformly distributed power allocation does not achieve the maximum of $E[\Phi(Q)]$.

\section{REFERENCES}

[1] G. J. Foschini and M. J. Gans, "On limits of wireless communications in a fading environment when using multiple antennas," Wireless Personal Commun. vol. 6, no.3, pp. 311-335, March 1998.

[2] I. E. Telatar, "Capacity of multi-antenna Gaussian channels," European Trans. on Telecommunications," vol. 10, no. 6, pp. 586-595, 1999.

[3] C. N. Chuah, D. Tse, J. M. Kahn and R. A. Valenzuela, "Capacity scaling in MIMO wireless systems under correlated fading," IEEE Trans. Inform. Theory, vol. 48, pp. 637-651, March 2002.

[4] G. G. Raleigh and V. K. Jones, "Multivariate modulation and coding for wireless communication," IEEE JSAC, pp. 851-866, May 1999.

[5] D. P. Palomar, J. M. Cioffi and M. A. Lagunas, "Uniform power allocation in MIMO channels: a game-theoretic approach," to appear in IEEE Trans. Inform. Theory, 2003.

[6] S. Verdú and H. V. Poor, "On minimax robustness: a general approach and applications," IEEE Trans. Inform. Theory, pp. 328-340, March 1984.

[7] R. A. Horn and C. R. Johnson, Matrix Analysis, Cambridge University Press, 1992. 